EUROPEAN JOURNAL OF PURE AND APPLIED MATHEMATICS

Vol. 12, No. 2, 2019, 348-357

ISSN 1307-5543 - www.ejpam.com

Published by New York Business Global

\title{
Common Fixed Points of Two Multivalued Asymptotically Nonexpansive Mappings
}

\author{
Safeer Hussain Khan ${ }^{1, *}$, Hira Iqbal ${ }^{2}$, Mujahid Abbas ${ }^{3,4}$ \\ ${ }^{1}$ Department of Mathematics, Statistics and Physics, Qatar University, Doha, 2713, Qatar \\ 2 Department of Sciences and Humanities, National University of Computer and Emerging \\ Sciences, Lahore Campus, Pakistan \\ ${ }^{3}$ Department of Mathematics, Government College University Lahore 54000, Pakistan \\ ${ }^{4}$ Department of Mathematics, King Abdulaziz University, P. O. Box. 80203, Jeddah 21589, \\ Saudi Arabia
}

\begin{abstract}
In this paper, we construct a modified Ishikawa iterative process to approximate common fixed points of two multivalued asymptotically nonexpansive mappings and prove some convergence theorems in uniformly convex hyperbolic spaces.
\end{abstract}

2010 Mathematics Subject Classifications: AMS 47H10, 47H09

Key Words and Phrases: Asymptotically nonexpansive mapping, multivalued mapping, common fixed point, Ishikawa iteration process

\section{Introduction}

Let $D$ be a nonempty subset of a metric space $(X, d)$. A mapping $T: D \rightarrow D$ is called asymptotically nonexpansive if for any $x, y \in D$, there exists a sequence $\left\{k_{n}\right\}$ with $k_{n} \geq 1$ and $\lim _{n \rightarrow \infty} k_{n}=1$ such that

$$
d\left(T^{n} x, T^{n} y\right) \leq k_{n} d(x, y) .
$$

Let $P(D)$ represent the set of all nonempty subsets of $D, C l(D)$ denote the set all nonempty closed subsets of $D$ and $C B(D)$ denote the set all nonempty closed and bounded subsets of $D$. For $A, B \in C B(D)$ and $x, y \in D$, define

$$
d(x, A)=\inf _{a \in A} d(x, a) .
$$

Then $H$ is known as the generalized Pompeiu-Hausdorff distance induced by $d$ if

$$
H(A, B)=\max \left\{\sup _{a \in A} d(a, B), \sup _{b \in B} d(A, b)\right\} .
$$

${ }^{*}$ Corresponding author.

DOI: https://doi.org/10.29020/nybg.ejpam.v12i2.3371

Email addresses: safeer@qu.edu.qa (S. H. Khan),

hira.iqbal@nu.edu.pk (H. Iqbal), abbas.mujahid@gmail.com (M. Abbas)

http://www.ejpam.com

348

(C) 2019 EJPAM All rights reserved. 
We say that a multivalued mapping $T: D \rightarrow P(D)$ has a fixed point $x$ if $x \in T x$. It is obvious that the theory of mutlivalued mappings is more complicated than that of the single valued mappings. Many different techniques have been employed to approximate fixed points of multivalued mappings. Rus [7] introduced the concept of generalized orbits in multivalued mappings. Khamsi and Kirk extended this concept of generalized orbits for the iterates of a multivalued mapping in [3].

Definition 1. Let $D$ be a nonempty subset of $X$ and $T: D \rightarrow P(D)$ be a multivalued mapping. We call $O(x, T)=\left\{x_{n}\right\}$ a generalized orbit of $x$ if for any $x \in D$ and $n \geq 0$, the sequence $\left\{x_{n}\right\}$ is defined by $x_{0}=x$ and $x_{n+1} \in T\left(x_{n}\right)$ where $n \in \mathbb{N} \cup\{0\}$.

Recently, in 2017, Khamsi and Khan [2], introduced the concept of a multivalued asymptotically nonexpansive mapping.

Definition 2. A mapping $T: D \rightarrow P(D)$ is called a multivalued asymptotically nonexpansive mapping if there exists a sequence $\left\{k_{n}\right\}$ with $k_{n} \geq 1$ and $\lim _{n \rightarrow \infty} k_{n}=1$ such that for any $x, y \in D$, and any generalized orbit $O(x, T)=\left\{x_{n}\right\}$ of $x$, there exists a generalized orbit $O(y, T)=\left\{y_{n}\right\}$ of $y$ such that

$$
d\left(x_{n+h}, y_{h}\right) \leq k_{h} d\left(x_{n}, y\right)
$$

where $n, h \in \mathbb{N}$.

They established the existence of a fixed point for a multivalued asymptotically nonexpansive mapping in hyperbolic metric spaces. They also proved the convergence of a modified Mann iterative process.

Motivated by this, we construct a modified Ishikawa iterative process for two multivalued asymptotically nonexpansive mappings and then prove some convergence theorems in uniformly convex hyperbolic metric spaces.

Next we recall the concept of hyperbolic metric spaces. Let $(X, d)$ be a metric space and $x, y$ be any two points in $X$. Then the unique metric segment $[x, y]$ is an isometric image of the real line interval $[0, d(x, y)]$. A point $z$ in $[x, y]$ is denoted as $\beta x \oplus(1-\beta) y$ which satisfies

$$
d(x, z)=(1-\beta) d(x, y) \quad \text { and } \quad d(z, y)=\beta d(x, y)
$$

where $\beta \in[0,1]$. Metric spaces with the class of metric segments are usually called convex metric spaces [5]. Further, if

$$
d(\alpha u \oplus(1-\alpha) x, \alpha v \oplus(1-\alpha) y) \leq \alpha d(u, v)+(1-\alpha) d(x, y)
$$

is satisfied for any $x, y, u, v \in X$ and $\alpha \in[0,1]$, then $X$ is said to be a hyperbolic metric space [6]. The following definition of uniformly convex hyperbolic metric space can be found in [1].

Definition 3. A hyperbolic metric space $(X, d)$ is called uniformly convex if for any $x, y, w \in X$, for every $r>0$, and for each $\epsilon>0$

$$
\delta(r, \epsilon)=\inf \left\{1-\frac{1}{r} d\left(\frac{1}{2} x \oplus \frac{1}{2} y, w\right)\right.
$$




$$
d(x, w) \leq r, d(y, w) \leq r, d(x, y) \geq r \epsilon\}>0 .
$$

Following is an important result in a uniformly convex hyperbolic metric space which will be used later.

Theorem 1. [4] Let $(X, d)$ be a uniformly convex complete hyperbolic metric space. Let $c>0$ and $z \in X$. Assume that $\left\{x^{n}\right\}$ and $\left\{y^{n}\right\}$ are two sequences in $X$ such that

$$
\begin{gathered}
\limsup _{n \rightarrow \infty} d\left(x^{n}, z\right) \leq c, \quad \limsup _{n \rightarrow \infty} d\left(y^{n}, z\right) \leq c, \\
\text { and, } \quad \lim _{n \rightarrow \infty} d\left(\alpha x^{n} \oplus(1-\alpha) y^{n}, z\right)=c
\end{gathered}
$$

then we have

$$
\lim _{n \rightarrow \infty} d\left(x^{n}, y^{n}\right)=0 .
$$

The following definition is needed in the sequel.

Definition 4. A multivalued mapping $T: D \rightarrow P(D)$ is said to be $H$-continuous if for any sequence $\left\{x^{n}\right\}$ which converges to $x$ in $D$, we have

$$
\lim _{n \rightarrow \infty} d\left(y^{n}, T(x)\right)=0
$$

for any sequence $\left\{y^{n}\right\}$ such that $y^{n} \in T x^{n}$ for any $n \in \mathbb{N}$.

Khamsi and Khan [2] have shown that every multivalued asymptotically nonexpansive mappings is $H$-continuous.

In this paper, we construct a modified Ishikawa iterative process which extends the Mann Type iterative process considered by Khamsi and Khan [2]. We use this iterative process to approximate common fixed points for two multivalued asymptotically nonexpansive mappings and prove some convergence theorems in uniformly convex hyperbolic spaces.

\section{Main Results}

Let $T_{1}$ and $T_{2}$ be two multivalued asymptotically nonexpansive mappings such that there exist sequences of positive numbers $\left\{k_{m}^{(1)}\right\}$ and $\left\{k_{m}^{(2)}\right\}$ with $k_{m}^{(i)} \in[1, \infty)$ and $\sum\left(k_{m}^{(i)}-\right.$ $1)<\infty$. Let

$$
k_{m}=\max \left\{k_{m}^{(1)}, k_{m}^{(2)}\right\},
$$

then $\sum\left(k_{m}-1\right)<\infty$. So, we take $\left\{k_{m}\right\}$ for both $T_{1}$ and $T_{2}$.

Now, we construct modified Ishikawa iterative process as follows:

Let $O\left(x^{1}, T_{1}\right)=\left\{x_{n}^{1}\right\}$ be a generalized orbit of $x^{1}$ associated with $T_{1}$ and $O\left(x^{1}, T_{2}\right)=$ $\left\{x_{n}^{1}\right\}$ be a generalized orbit of $x^{1}$ associated with $T_{2}$. Fix $0<\alpha, \beta<1$ and set

$$
x^{2}=\alpha x^{1} \oplus(1-\alpha) y_{1}^{1},
$$




$$
y^{1}=\beta x^{1} \oplus(1-\beta) x_{1}^{1} .
$$

Again, let $O\left(x^{2}, T_{1}\right)=\left\{x_{n}^{2}\right\}$ be a generalized orbit of $x^{2}$ associated with $T_{1}$ and $O\left(x^{2}, T_{2}\right)=$ $\left\{x_{n}^{2}\right\}$ be a generalized orbit of $x^{2}$ associated with $T_{2}$ where

$$
\begin{aligned}
d\left(x_{n+h}^{1}, x_{h}^{2}\right) & \leq k_{h} d\left(x_{n}^{1}, x^{2}\right), \\
\text { and } \quad d\left(x_{n+h}^{1} 1^{\prime}, x_{h}^{2}\right) & \leq k_{h} d\left(x_{n}^{1}, x^{2}\right) .
\end{aligned}
$$

Hence, using induction for any $m \geq 1$ we have a sequence $\left\{x^{m}\right\}$ in $D$ and the orbits $O\left(x^{m}, T_{1}\right)=\left\{x_{n}^{m}\right\}$ and $O\left(x^{m}, T_{2}\right)=\left\{x_{n}^{m}\right\}$ such that

$$
\begin{aligned}
x^{m+1} & =\alpha x^{m} \oplus(1-\alpha) y_{m}^{m}, \\
y^{m} & =\beta x^{m} \oplus(1-\beta) x_{m}^{m}
\end{aligned}
$$

and

$$
\begin{aligned}
d\left(x_{n+h}^{m-1}, x_{h}^{m}\right) & \leq k_{h} d\left(x_{n}^{m-1}, x^{m}\right), \\
\text { and } \quad d\left(x_{n+h}^{\prime \prime}, x_{h}^{m}\right) & \leq k_{h} d\left(x_{n}^{m^{\prime}-1}, x^{m}\right) .
\end{aligned}
$$

Throughout this section, we denote $F=F\left(T_{1}\right) \cap F\left(T_{2}\right)$.

Lemma 1. Let $(X, d)$ be a complete uniformly convex hyperbolic metric space. Let D be a nonempty bounded, closed and convex subset of $X$. Let $T_{1}$ and $T_{2}$ be multivalued asymptotically nonexpansive mappings with $k_{m} \in[1, \infty)$ and $\sum_{m=1}^{\infty}\left(k_{m}-1\right)<\infty$. Define the sequence as in (1). If

$$
\lim _{m \rightarrow \infty} d\left(x^{m}, x_{m}^{m}\right)=0=\lim _{m \rightarrow \infty} d\left(x^{m}, \dot{x}_{m}^{m}\right),
$$

then

$$
\lim _{m \rightarrow \infty} d\left(x^{m}, x_{1}^{m}\right)=0=\lim _{m \rightarrow \infty} d\left(x^{m}, \dot{x}_{1}^{m}\right) .
$$

Proof. Let

$$
d\left(x^{m}, x_{m}^{m}\right)=a_{m}, \quad \text { and } \quad d\left(x^{m}, x_{m}^{m}\right)=b_{m} .
$$

Since $x^{m+1}=\alpha x^{m} \oplus(1-\alpha) y_{m}^{m}$, we have

$$
\begin{aligned}
d\left(x^{m+1}, x^{m}\right) & \leq(1-\alpha) d\left(x^{m}, \dot{y}_{m}^{m}\right) \\
& \leq d\left(x^{m}, \dot{y}_{m}^{m}\right) \\
& \leq d\left(x^{m}, \dot{x}_{m}^{m}\right)+d\left(\dot{x}_{m}^{m}, \dot{y}_{m}^{m}\right) \\
& \leq a_{m}+d\left(\dot{x}_{m}^{m}, \dot{x}_{m+m}^{m}\right)+d\left(\dot{x}_{m+m}^{m}, \dot{y}_{m}^{m}\right) \\
& \leq a_{m}+k_{m} d\left(\dot{x}_{m}^{m}, x^{m}\right)+k_{m} d\left(\dot{x}_{m}^{m}, y^{m}\right) \\
& \leq a_{m}+k_{m} a_{m}+k_{m}\left(\beta d\left(\dot{x}_{m}^{m}, x^{m}\right)\right.
\end{aligned}
$$


S. H. Khan, H. Iqbal, M. Abbas / Eur. J. Pure Appl. Math, 12 (2) (2019), 348-357

$$
\begin{aligned}
& \left.+(1-\beta) d\left(\dot{x}_{m}^{m}, x_{m}^{m}\right)\right) \\
\leq \quad & a_{m}+k_{m} a_{m}+k_{m} \beta a_{m}+k_{m}(1-\beta) d\left(\dot{x}_{m}^{m}, x^{m}\right) \\
& +k_{m}(1-\beta) d\left(x_{m}^{m}, x^{m}\right) \\
\leq \quad & a_{m}+k_{m} a_{m}+k_{m} \beta a_{m} \\
& +k_{m}(1-\beta) a_{m}+k_{m}(1-\beta) b_{m} \\
\leq \quad & \left(1+2 k_{m}\right) a_{m}+k_{m} b_{m} .
\end{aligned}
$$

Taking $\lim _{m \rightarrow \infty}$ in the above inequality, we get

$$
\lim _{m \rightarrow \infty} d\left(x^{m+1}, x^{m}\right) \leq \lim _{m \rightarrow \infty}\left(1+2 k_{m}\right) a_{m}+\lim _{m \rightarrow \infty} k_{m} b_{m}=0 .
$$

That is

$$
\lim _{m \rightarrow \infty} d\left(x^{m+1}, x^{m}\right)=0
$$

Moreover, from (2)

$$
\begin{aligned}
d\left(x^{m+1}, x_{1}^{m^{\prime}+1}\right) & \leq d\left(x^{m+1}, x_{m+1}^{m^{\prime}+1}\right)+d\left(x_{m+1}^{m^{\prime}+1}, x_{1}^{m^{\prime}+1}\right), \\
& \leq a_{m+1}+k_{1} d\left(x^{m+1}, x_{m}^{m+1}\right), \\
& \leq a_{m+1}+k_{1}\left[d\left(x^{m}, x^{m+1}\right)+d\left(x^{m}, x_{m}^{m}\right)+d\left(x_{m}^{m}, x_{m}^{m^{\prime}+1}\right)\right], \\
& \leq a_{m+1}+k_{1}\left[d\left(x^{m}, x^{m+1}\right)+d\left(x^{m}, x_{m}^{m}\right)+k_{m} d\left(x^{m}, x^{m+1}\right)\right], \\
& =a_{m+1}+k_{1}\left[a_{m}+\left(1+k_{m}\right) d\left(x^{m}, x^{m+1}\right)\right] .
\end{aligned}
$$

Then

$$
\begin{aligned}
\lim _{m \rightarrow \infty} d\left(x^{m+1}, x_{1}^{m+1}\right) & \leq \lim _{m \rightarrow \infty} a_{m+1}+k_{1} \lim _{m \rightarrow \infty}\left[a_{m}+\left(1+k_{m}\right) d\left(x^{m}, x^{m+1}\right)\right] \\
& =0
\end{aligned}
$$

Hence

$$
\lim _{m \rightarrow \infty} d\left(x^{m}, x_{1}^{m}\right)=0
$$

Similarly

$$
\begin{aligned}
d\left(x^{m+1}, x_{1}^{m+1}\right) & \leq d\left(x^{m+1}, x_{m+1}^{m+1}\right)+d\left(x_{m+1}^{m+1}, x_{1}^{m+1}\right) \\
& \leq b_{m+1}+k_{1} d\left(x^{m+1}, x_{m}^{m+1}\right) \\
& \leq b_{m+1}+k_{1}\left[d\left(x^{m}, x_{m+1)}+d\left(x^{m}, x_{m}^{m}\right)+d\left(x_{m}^{m}, x_{m}^{m+1}\right)\right]\right. \\
& \leq b_{m+1}+k_{1}\left[d\left(x^{m}, x^{m+1}\right)+d\left(x^{m}, x_{m}^{m}\right)+k_{m} d\left(x^{m}, x^{m+1}\right)\right] \\
& =b_{m+1}+k_{1}\left[b_{m}+\left(1+k_{m}\right) d\left(x^{m}, x^{m+1}\right)\right] .
\end{aligned}
$$

Consequently

$$
\lim _{m \rightarrow \infty} d\left(x^{m}, x_{1}^{m}\right)=0 .
$$


Theorem 2. Let $(X, d)$ be a complete uniformly convex hyperbolic metric space. Let $D$ be a nonempty bounded, closed and convex subset of $X$. Let $T_{1}$ and $T_{2}$ be multivalued asymptotically nonexpansive mappings. Let $k_{m}$ be the Lipschitz sequence associated with $T_{1}$ and $T_{2}$ such that $k_{m} \in[1, \infty]$ and $\sum_{m=1}^{\infty}\left(k_{m}-1\right)<\infty$. Let $F \neq \varnothing$ and $T_{1} p=T_{2} p=\{p\}$ for $p \in F$. Fix $x_{1} \in D$ and $\alpha \in(0, \infty)$. Suppose $x^{m}$ is defined as in (1). Then,

$$
\lim _{m \rightarrow \infty} d\left(x^{m}, x_{1}^{m}\right)=0, \quad \text { and } \quad \lim _{m \rightarrow \infty} d\left(x^{m}, \dot{x}_{1}^{m}\right)=0 .
$$

That is,

$$
\lim _{m \rightarrow \infty} d\left(x^{m}, T_{1} x^{m}\right)=0, \quad \text { and } \quad \lim _{m \rightarrow \infty} d\left(x^{m}, T_{2} x^{m}\right)=0 .
$$

Proof. Let $p \in D$ be such that $p \in F$ and $T_{1} p=T_{2} p=\{p\}$. Then

$$
\text { and } \begin{aligned}
d\left(x_{n+h}^{m}, p\right) & \leq k_{h} d\left(x_{n}^{m}, p\right) \\
\text { and } & \left.x_{n+h}^{m}, p\right) \leq k_{h} d\left(x_{n}^{m}, p\right) .
\end{aligned}
$$

Now

$$
\begin{aligned}
d\left(x^{m+1}, p\right) & \leq \alpha d\left(x^{m}, p\right)+(1-\alpha) d\left(y_{m}^{m}, p\right) \\
& \leq \alpha d\left(x^{m}, p\right)+(1-\alpha) k_{m} d\left(y^{m}, p\right)
\end{aligned}
$$

and

$$
\begin{aligned}
d\left(y^{m}, p\right) & \leq \beta d\left(x^{m}, p\right)+(1-\beta) d\left(x_{m}^{m}, p\right) \\
& \leq \beta d\left(x^{m}, p\right)+(1-\beta) k_{m} d\left(x^{m}, p\right) .
\end{aligned}
$$

Inequalities (3) and (4) imply,

$$
\begin{aligned}
d\left(x^{m+1}, p\right) & \leq \alpha d\left(x^{m}, p\right)+(1-\alpha) k_{m}\left[\beta+(1-\beta) k_{m}\right] d\left(x^{m}, p\right) \\
& =\left[\alpha+\beta(1-\alpha) k_{m}+(1-\beta)(1-\alpha) k_{m}^{2}\right] d\left(x^{m}, p\right) \\
& =V_{m} d\left(x^{m}, p\right)
\end{aligned}
$$

where $V_{m}=\alpha+\beta(1-\alpha) k_{m}+(1-\beta)(1-\alpha) k_{m}^{2}$.

This implies,

$$
d\left(x^{m+1}, p\right)-d\left(x^{m}, p\right) \leq\left(V_{m}-1\right) d\left(x^{m}, p\right) \leq\left(V_{m}-1\right) \delta(D)
$$

for any $m \in \mathbb{N}$, where $\delta(D)=\sup _{x, y \in D}\{d(x, y)\}$ is the diameter of $D$. Therefore,

$$
d\left(x^{m+h}, p\right)-d\left(x^{m}, p\right) \leq \sum_{i=m}^{m+(h-1)}\left(V_{i}-1\right) \delta(D) .
$$

Since $\sum_{m=1}^{\infty}\left(k_{m}-1\right)<\infty$ so $\sum_{m=1}^{\infty}\left(V_{m}-1\right)<\infty$. Let $h \rightarrow \infty$,

$$
\limsup _{n \rightarrow \infty} d\left(x^{n}, p\right)-d\left(x^{m}, p\right) \leq \delta(D) \sum_{i=m}^{\infty}\left(V_{i}-1\right),
$$


for any $m \in \mathbb{N}$. Now letting $m \rightarrow \infty$,

$$
\limsup _{n \rightarrow \infty} d\left(x^{n}, p\right) \leq \liminf _{m \rightarrow \infty} d\left(x^{m}, p\right)
$$

This implies $\left\{d\left(x^{n}, p\right)\right\}$ is convergent. Let

$$
c=\lim _{n \rightarrow \infty} d\left(x^{n}, p\right)
$$

If $c=0$, then we have nothing to prove. So we take $c>0$. Since $d\left(x_{n}^{n}, p\right) \leq k_{n} d\left(x^{n}, p\right)$,

$$
\limsup _{n \rightarrow \infty} d\left(x_{n}^{n}, p\right) \leq c
$$

Also,

$$
\limsup _{n \rightarrow \infty} d\left(y^{n}, p\right) \leq c .
$$

We know that if $\lim _{n \rightarrow \infty} k_{n}=1$ then $\lim _{n \rightarrow \infty} k_{n}^{2}=1$, therefore,

$$
\begin{aligned}
\limsup _{n \rightarrow \infty} d\left(\dot{y}_{n}^{n}, p\right) & \leq \limsup _{n \rightarrow \infty} k_{n} d\left(y^{n}, p\right) \\
& \leq \limsup _{n \rightarrow \infty} k_{n}\left[\beta d\left(x^{n}, p\right)+(1-\beta) d\left(x_{n}^{n}, p\right)\right] \\
& \leq \limsup _{n \rightarrow \infty} k_{n}\left[\beta d\left(x^{n}, p\right)+(1-\beta) k_{n} d\left(x^{n}, p\right)\right] \\
& =c,
\end{aligned}
$$

and

$$
c=\lim _{n \rightarrow \infty} d\left(x^{n+1}, p\right)=\lim _{n \rightarrow \infty} d\left(\alpha x^{n} \oplus(1-\alpha) y_{n}^{n}, p\right) .
$$

Then, from Theorem $1,(5),(7)$ and (8), we get

$$
\lim _{n \rightarrow \infty} d\left(x^{n}, y_{n}^{n}\right)=0
$$

Next

$$
\begin{aligned}
d\left(x^{n}, p\right) & \leq d\left(x^{n}, y_{n}^{n}\right)+d\left(y_{n}^{n}, p\right) \\
& \leq d\left(x^{n}, y_{n}^{n}\right)+k_{n} d\left(p, y^{n}\right)
\end{aligned}
$$

implies

$$
\liminf _{n \rightarrow \infty} d\left(x^{n}, p\right) \leq \liminf _{n \rightarrow \infty}\left(d\left(x^{n}, y_{n}^{n}\right)+k_{n} d\left(p, y^{n}\right)\right) .
$$

Using (5) and (9), we get

$$
c \leq \liminf _{n \rightarrow \infty} d\left(y^{n}, p\right) .
$$

Then (7) and (10) imply

$$
\lim _{n \rightarrow \infty} d\left(y^{n}, p\right)=c
$$


That is,

$$
c=\lim _{n \rightarrow \infty} d\left(y^{n}, p\right)=\lim _{n \rightarrow \infty} d\left(\beta x^{n} \oplus(1-\beta) x_{n}^{n}\right) .
$$

From Theorem 1, (5), (6) and (12), we get,

$$
d\left(x^{n}, x_{n}^{n}\right)=0 .
$$

Also,

$$
\begin{aligned}
d\left(x^{n}, x_{n}^{\prime}\right) & \leq d\left(x^{n}, y_{n}^{n}\right)+d\left(y_{n}^{n}, x_{n}^{n}\right) \\
& \leq d\left(x^{n}, y_{n}^{n}\right)+k_{n} d\left(x^{n}, y^{n}\right) \\
& \leq d\left(x^{n}, y_{n}^{n}\right)+k_{n}(1-\alpha) d\left(x^{n}, x_{n}^{n}\right)
\end{aligned}
$$

Hence, (9) and (13) give

$$
\lim _{n \rightarrow \infty} d\left(x^{n}, x_{n}^{\prime n}\right)=0 .
$$

Now using Lemma 1, (13) and (14), we get our desired results.

We now give some convergence results.

Theorem 3. Let $D$ be a compact and convex subset of a uniformly convex hyperbolic space. Let $T_{1}, T_{2}$ and $x^{n}$ be as in Theorem 2. If $F \neq \varnothing$ with $T_{1} p=T_{2} p=\{p\}$ for $p \in F$ then there is a subsequence of $\left\{x^{n}\right\}$ which converges to a common fixed point of $T_{1}$ and $T_{2}$.

Proof. Since $D$ is compact so there exists a subsequence $\left\{x^{n_{k}}\right\}$ of $\left\{x^{n}\right\}$ such that $\left\{x^{n_{k}}\right\}$ converges to some $z \in D$. From Theorem 2 we know that

$$
\lim _{m \rightarrow \infty} d\left(x^{n}, x_{1}^{n}\right)=0, \quad \text { and } \quad \lim _{n \rightarrow \infty} d\left(x^{n}, \dot{x}_{1}^{n}\right)=0 .
$$

Applying $H$-continuity of $T_{1}$ and $T_{2}$, we have

$$
\lim _{n \rightarrow \infty} d\left(T z, x_{1}^{n_{k}}\right)=\lim _{n \rightarrow \infty} d\left(T_{1} z, x_{1}^{n_{k}}\right)=0
$$

where $x_{1}^{n_{k}} \in T_{1} x^{n_{k}}$ and $x_{1}^{n_{k}} \in T_{2} x^{n_{k}}$. Thus

$$
d\left(z, T_{1} z\right) \leq d\left(T_{1} z, x_{1}^{n_{k}}\right)+d\left(z, x^{n_{k}}\right)+d\left(x_{1}^{n_{k}}, x^{n_{k}}\right) .
$$

Therefore, as $n \rightarrow \infty$

$$
d\left(z, T_{1} z\right)=0 .
$$

Similarly,

$$
d\left(z, T_{2} z\right) \leq d\left(T_{2} z, x_{1}^{\dot{n}_{k}}\right)+d\left(z, x^{n_{k}}\right)+d\left(x_{1}^{\hat{n}_{k}}, x^{n_{k}}\right) .
$$

Thus, as $n \rightarrow \infty$,

$$
d\left(z, T_{2} z\right)=0 .
$$

Hence, $z$ is a common fixed point of $T_{1}$ and $T_{2}$. 
Theorem 4. Let $D$ be a nonempty, closed, convex and bounded subset of a complete uniformly convex hyperbolic space $(X, d)$. Let $T_{1}, T_{2}$ and $x^{n}$ be as in Theorem 2. If $F \neq \varnothing$ with $T_{1} p=T_{2} p=\{p\}$ for $p \in F$, then $\left\{x^{n}\right\}$ converges to a common fixed point of $T_{1}$ and $T_{2}$ if and only if $\liminf _{n \rightarrow \infty} d\left(x^{n}, F\right)=0$ where $d\left(x^{n}, F\right)=\inf \left\{d\left(x^{n}, p\right): p \in F\right\}$.

Proof. The necessity of the conditions is obvious. Conversely, suppose that $\liminf _{n \rightarrow \infty} d\left(x^{n}, F\right)=$ 0. Since,

$$
\begin{aligned}
d\left(x^{n+1}, p\right) & \leq V_{n} d\left(x^{n}, p\right) \\
\Rightarrow d\left(x^{n+1}, F\right) & \leq V_{n} d\left(x^{n}, F\right) .
\end{aligned}
$$

Thus, $\lim _{n \rightarrow \infty}\left(x^{n}, F\right)$ exists.

Since $\liminf \operatorname{in}_{n \rightarrow \infty} d\left(x^{n}, F\right)=0, \lim _{n \rightarrow \infty}\left(x^{n}, F\right)=0$. Next, from $1+x \leq e^{x}$ for all $x \geq 0$, we obtain

$$
\begin{aligned}
d\left(x^{n+k}, p\right) & \leq V_{n+(k-1)} d\left(x^{n+(k-1)}, p\right) \\
& =\left(1+\left(V_{n+(k-1)}-1\right)\right) d\left(x^{n+(k-1)}, p\right) \\
& \leq e^{V_{n+(k-1)}-1} d\left(x^{n+(k-1)}, p\right) \\
& \leq e^{V_{n+(k-1)}-1} e^{V_{n+(k-2)}-1} d\left(x^{n+(k-2)}, p\right) \\
& \leq: \quad: \quad: \\
& \leq: \quad: \quad: \\
& \leq e^{\sum_{i=n}^{n+(k-1)}\left(V_{i}-1\right)} d\left(x^{n}, p\right) .
\end{aligned}
$$

We know that $\sum_{n}\left(V_{n}-1\right)<\infty$,so there exists some $W$ such that,

$$
d\left(x^{n+k}, p\right) \leq W d\left(x^{n}, p\right)
$$

for all $p \in F$ and $n \in \mathbb{N}$. Since $\lim _{n \rightarrow \infty} d\left(x^{n}, F\right)=0, \exists n_{0}$ such that

$$
d\left(x^{n_{0}}, F\right)<\frac{\epsilon}{W+1} .
$$

Thus there must exist $p^{*}$ such that

$$
d\left(x^{n_{0}}, p^{*}\right)<\frac{\epsilon}{W+1} .
$$

Hence

$$
\begin{aligned}
d\left(x^{n_{0}+k}, x^{n_{0}}\right) & \leq d\left(x^{n_{0}+k}, p^{*}\right)+d\left(p^{*}, x^{n_{0}}\right) \\
& \leq W d\left(p^{*}, x^{n_{0}}\right)+d\left(p^{*}, x^{n_{0}}\right) \\
& <W\left(\frac{\epsilon}{W+1}\right)+\frac{\epsilon}{W+1} \\
& =\epsilon
\end{aligned}
$$


This shows that $\left\{x^{n}\right\}$ is Cauchy in $D$. Since $D$ is closed, $x^{n}$ converges to some $z$ in $D$. Next, we show that $z \in F$. Let $d\left(z, x^{n}\right)<\frac{\epsilon}{4\left(k_{1}+1\right)}$ and let $z_{*} \in F$. Then $d\left(x^{n}, z_{*}\right)<\frac{\epsilon}{4\left(k_{1}+1\right)}$ and $d\left(z_{*}, T z\right)<\frac{\epsilon}{2\left(k_{1}+1\right)}$ so that

$$
d\left(z, z_{*}\right)<d\left(z, x^{n}\right)+d\left(x^{n}, z_{*}\right)<\frac{\epsilon}{2\left(k_{1}+1\right)} .
$$

Finally,

$$
\begin{aligned}
d\left(z, T_{1} z\right) & \leq d\left(z, x^{n}\right)+d\left(x^{n}, z_{*}\right)+d\left(z_{*}, T_{1} z\right) \\
& \leq d\left(z, x^{n}\right)+d\left(x^{n}, z_{*}\right)+k_{1} d\left(z_{*}, z\right) \\
& <\frac{\epsilon}{4\left(k_{1}+1\right)}+\frac{\epsilon}{4\left(k_{1}+1\right)}+\frac{\epsilon}{2\left(k_{1}+1\right)} \\
& <\epsilon .
\end{aligned}
$$

Therefore $z \in T_{1} z$. Similarly $z \in T_{2} z$.

Hence $T_{1}$ and $T_{2}$ have a common fixed point.

\section{References}

[1] K Goebel and W A Kirk. Topics in metric fixed point theory. Cambridge University Press, 1990.

[2] M A Khamsi and A R Khan. Goebel and Kirk fixed point theorem for multivalued symptotically nonexpansive mappings. Carpathian Journal of Mathematics, 33(3):335$342,2017$.

[3] M A Khamsi and W A Kirk. On Uniformly Lipschitzian Multivalued Mappings in Banach and Metric spaces. Nonlinear Analysis, 72:2080-2085, 2010.

[4] A R Khan and M A A Khan. An implicit algorithm for two finite families of nonexpansive maps in hyperbolic spaces. Fixed Point Theory and Applications, 2012:54, 2012 .

[5] K Menger. Untersuchungen über allgemeine Metrik. Mathematische Annalen, 100:75$163,1928$.

[6] S Reich and I Shafrir. Nonexpansive iterations in hyperbolic spaces. Nonlinear Analysis, 15:537-558, 1990.

[7] I A Rus. Basic problems of the metric fixed point theory revisited, II. Studia Universitatis Babe-Bolyai Mathematica, 36:81-99, 1991. 